\title{
Intelligent Data Analysis: Keeping Pace with Technological Advances
}

\author{
Xiaohui Liu \\ School of Information Systems, Computing and Mathematics, \\ Brunel University, London UB8 3PH, UK \\ Xiaohui.Liu@brunel.ac.uk
}

\begin{abstract}
Over the past few decades, we have witnessed significant advances in technology that have done so much to change the way we live and communicate, e.g. the medical and biotechnology, the internet and mobile technology etc. Often these technologies lead to a huge amount of data being generated, and making best use of these technologies often depends on how best to interpret these data in the context of many problem solving and complex systems. Intelligent Data Analysis is needed to address the interdisciplinary challenges concerned with the effective analysis of data [1-3]. In this talk, I will look into a range of real world complex systems via technological changes and explore the role of IDA in these systems, in particular, how to ensure that quality data are obtained for analysis, to handle human factors and domain knowledge with care, to meet challenges in modelling dynamic systems, as well as to consider all these when analysing complex systems [4-10].
\end{abstract}

\section{References}

1. Liu, X.: Intelligent Data Analysis: Issues and Challenges. The Knowledge Engineering Review 11(4), 365-371 (1996)

2. Berthold, M., Hand, D.J.: Intelligent Data Analysis: an Introduction, 2nd edn. Springer, Heidelberg (2007)

3. Cohen, P., Adams, N.: Intelligent Data Analysis in the 21st Century. In: Adams, N.M., Robardet, C., Siebes, A., Boulicaut, J.-F. (eds.) IDA 2009. LNCS, vol. 5772, pp. 1-9. Springer, Heidelberg (2009)

4. Liu, X., Cheng, G., Wu, J.: Analysing Outliers Cautiously. IEEE Transactions on Knowledge and Data Engineering 14, 432-437 (2002)

5. Swift, S., Tucker, A., Vinciotti, V., Martin, M., Orengo, C., Liu, X., Kellam, P.: Consensus Clustering and Functional Interpretation of Gene Expression Data. Genome Biology 5, R94 (2004)

6. Chen, S., Liu, X.: An Integrated Approach for Modeling Learning Patterns of Students in Web-Based Instruction: A Cognitive Style Perspective. ACM Transactions on Computer Human Interaction 15(1), 1-28 (2008)

7. Wang, Z., Liu, X., Liu, Y., Liang, J., Vinciotti, V.: An Extended Kalman Filtering Approach to Modelling Nonlinear Dynamic Gene Regulatory Networks. IEEE/ACM Transactions on Computational Biology and Bioinformatics 6(3), 410-419 (2009)

8. Ruta, A., Li, Y., Liu, X.: Real-Time Traffic Sign Recognition from Video by ClassSpecific Discriminative Features. Pattern Recognition 43(1), 416-430 (2010)

9. Fraser, K., Wang, Z., Liu, X.: Microarray Image Analysis: an Algorithmic Approach. Chapman \& Hall/CRC, London (2010)

10. Liang, J., Wang, Z., Liu, X.: Distributed State Estimation for Discrete-Time Sensor Networks with Randomly Varying Nonlinearities and Missing Measurements. IEEE Transactions on Neural Networks 22(3), 486-496 (2011) 